\title{
Performance Impact of Relay Selection in WiMAX IEEE 802.16j Multi-hop Relay Networks
}

\author{
Noman Mazhar ${ }^{1}$ \\ Faculty of Computer Science and Information Technology \\ University of Malaya \\ Kuala Lumpur, Malaysia
}

\author{
Muhammad Zeeshan ${ }^{2}$, Anjum Naveed ${ }^{3}$ \\ School of Electrical Engineering and Computer Science \\ National University of Sciences and Technology (NUST) \\ Islamabad, Pakistan
}

\begin{abstract}
Worldwide Interoperability for Microwave Access network accepts the challenge of last mile wireless access for internet. IEEE 802.16 standard, commercially known as WiMAX provide wireless broadband experience to the end subscribers and challenges many wired solutions like Digital Subscriber Line (DSL) and cable internet. Wireless network has many inherent issues like coverage holes; capacity optimization and mobility are few of them. Adding relays to multi-hop WiMAX IEEE 802.16j network present an effective solution to address them to some extent but this amendment does not elaborate any algorithm regarding the relay selection and narrate no performance guarantees. In this work, we proposed linear model that fairly allocates wireless resources among subscribers in 802.16j network. A relay selection algorithm is also presented to optimally select nodes with higher signal-to-noise ratio as relay station for nodes with lower signal-to-noise ratio objectively maximize overall network capacity. This scheme further extends network coverage area and improves network availability. We also did extensive performance evaluation of the proposed linear model. Results show that optimal relays selection scheme do provide a substantial increase of up to $66 \%$ in overall network capacity in the fixed WiMAX network. This improvement is substantial at places where network condition is not optimal. Investigating the problem further leads to the conclusion that the relay selection criterion is the key to achieve maximum network capacity.
\end{abstract} SNR

Keywords-WiMAX; multi-hop; wireless broadband; relay;

\section{INTRODUCTION}

High speed internet access for the last mile has been a challenge over the years. Apart from the wired technology, inception of advanced coding schemes and antenna technology made wireless broadband a competitive solution. IEEE 802.16 Working Group (2004) commercially known as WiMAX initially formalized in 2001, however till 2004 standard based on [1] targeted only fixed applications and the standard was referred to these as fixed WiMAX. In December 2005 new amendment IEEE 802.16e [2] was launched which added a new dimension in WiMAX, mobility. Since then IEEE 802.16e-2005 forms the basis for the WiMAX solution for nomadic and mobile applications and is often referred to as mobile WiMAX. IEEE 802.16 [3] merges the fixed and mobile PHY and MAC capabilities of the network. Another amendment IEEE 802.16 [4] modified the physical layer and the MAC layer for inclusion of relays in the network known as IEEE 802.16j. The current version of IEEE 802.16 [5] has added further modification to support higher reliability networks.

Multi-hop wireless network started to get much focus in the telecommunication industry, due to rapid deployment and coverage enhancements. This also forces various product portfolios to upgrade to this technology in order to get major share of the industry in near future. There are many networks which provide multi-hop communication, but all of these have different MAC and PHY layer design due to constraint like power, mobility and transmission range. Augmenting to this, multi-hop wireless networks require complex routing algorithms. All this added to the limited use of the multi-hop networks. Introduction of relays in multi-hop wireless network have improved the network capacity and extend coverage area and simplify deployment scenario. According to Pabst R. et al. [6], relays provide communication to the nodes outside transmission range of the base station (BS), support in alleviating the range limitation for wireless broadband networks.

There are many practical scenarios as shown in the Fig. 1, where relays do play important role. Relays can be placed in different formations to enhance the system performance and coverage. In fixed infrastructure the relays are placed in stationary areas by the service provider to provide normal traffic by extenuating the loop holes and extending the coverage.

To provide relay based multi-hop support in WiMAX, a new amendment to the standard is made known as IEEE $802.16 \mathrm{j}$ and it defines two types of relays transparent and nontransparent relays. Transparent relays are used for improvement in capacity of overall network while the use of non-transparent relays extends the coverage area. The major difference between the two relay modes is the way framing information is transmitted. In transparent mode frame header information is not transmitted; while in non-transparent mode the frame contains the header information. The frame header encapsulates critical scheduling information which the nodes used to determine when it can transmit or receive. In relay modes there are two types of scheduling modes: centralized and distributed. Base Station (BS) control scheduling for all the nodes in centralized mode while in distributed mode relay station (RS) can take some scheduling decision for the nodes attach to it. But there are challenges faced by the system implementing $802.16 \mathrm{j}$ like frequency reuse, resource allocation, relay selection, performance and scheduling. IEEE 
802.16j standard has enhanced BS and RS capabilities to meet multihop networks communication challenges. The standard does not discuss much about relay selection criteria. This issue has been left vendor specific. Similarly, no performance parameters are given while using relays in the WiMAX network.

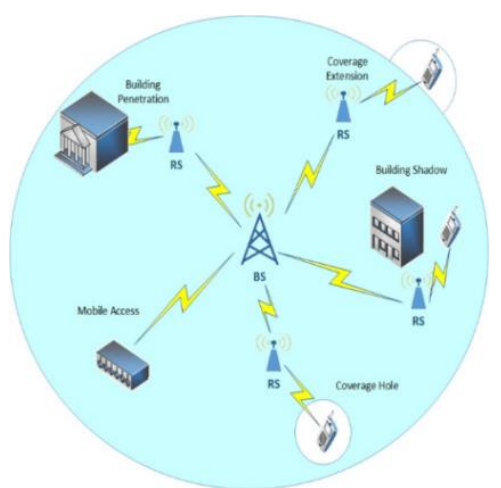

Fig. 1. Relay Application.

In this study we focus on the relay selection such that throughput of overall network is optimized in IEEE 802.16j network. In this context we propose linear model that fairly allocates the resources among subscribers in the range of BS. In fixed and nomadic infrastructure relays with centralized scheduling is the simplest and cost-effective solution to the multi-hop relay network. However distributed scheduling mode is more affective for mobile applications in order to handle coverage extension and other mobility issues. Therefore, the study focuses on centralized scheduling in fixed WiMAX network. We develop linear model using linear programming technique. We implemented the optimization model using algebraic modeling problem language (AMPL) and use simplex method for testing its results. We also develop a simulation program in $\mathrm{c}$ programing language to test the system model against IEEE 802.16-2004 based network without relays. Results show considerable throughput enhancement as compare to IEEE 802.16-2004. Further in this research we consider two main aspects: (i) relay selection scheme in IEEE 802.16j network, (ii) implications of relays on the overall network performance. Followings are the contributions of this work:

- We first propose a linear model to evaluate the performance of WiMAX network.

- We also develop relays selection algorithm for WiMAX multi-hop network and perform optimization of proposed linear model.

- We then perform comprehensive performance analysis of capacity gains after inclusion of relays in the WiMAX multi-hop network.

- Results show that proposed relay-based model out performs traditional WiMAX network in terms of capacity utilization.

The rest of the paper is organized as follows. In Section 2, related work has been elaborated. Section 3 presents the system model and problem formulation. Section 4 discusses the relay selection algorithm. The results and analysis of proposed algorithm are detailed in Section 5. Section 6 concludes this work.

\section{RELATED WORK}

In recent years, some research tried to predict the performance of IEEE $802.16 \mathrm{j}$ network under different realizations and addressed issues like performance of network with inclusion of relays. Other issues include handling the loop holes and actual coverage extension.

In recent work on the performance evaluation of the WiMAX network, there are different attributes of wireless network that are exploited to enhance the network throughput. Like the work of Bonald T. et al. [10] is to determine the maximum throughput gain achievable under max-min fairness in which approximately equal performance is delivered to all subscribers. Like the Genc V. et al. [16] present an analytical modal that tries to enhance end-to-end throughput under maxmin fairness constraint. Further by Genc V. et al. [17] extends the same model by incorporating the variable number of relays and transmits power; results show that about $55 \%$ to $125 \%$ of the throughput gain can be achieved subject to spatial reuse. But the MAXMIN algorithm has an issue that it may increase the throughput of some subscribers but starved the rest of the nodes. Another way is to compare the SNR for each path as done by Shrestha D. M. et al. [11] purposes more advance technique which use backward compatible signaling mechanism and introduce a centralize path selection algorithm based on ELT (estimated link throughput). ELT is based on available bandwidth and data rate for maximum throughput where data rate depends on SNR. ELT calculation for each path and signaling make base station processing more complex which compromises the overall performance of the network.

Similar work is purposed by Ann S. et al. [14] tries to find the route that reduces the latency and maximizes the network throughput. It is a centralized algorithm that makes decision based on SNR, available bandwidth, hop count but this scheme does uneven resource allocation and starves many subscribers. WiMAX provides two modes centralized and distributed scheduling. Some research uses centralized mode to achieve the performance as in $\mathrm{Li} \mathrm{D}$. et al. [18] focus on the maximum throughput in two hop fixed WiMAX network by using centralized scheduling scheme. Relay selection algorithm in such proposal is based on quality-of-service QoS, parameters including SNR and latency. But it did not warrant throughput maximization that can be achieved using this algorithm. One of the techniques is to measure the resource cost for the path, in order to select the most optimum path for the channel like the work in [20] proposes a path selection model based on radio resource cost (RRC) and the minimum the cost the best is the path. The result shows the throughput obtain using RRC scheme is much better as compare to the rest of scenarios. An interesting study use the adaptive technique according to the channel conditions as in Chang J. Y. et al. in [22] develop a deployment algorithm that work based on traffic and uniform clustering. This study takes both RS and BS placement to get the best throughput and coverage. The algorithm provides the vendors an adaptive deployment of $\mathrm{BS}$ and $\mathrm{RS}$, further considering the environment. 
Liu I. H. et al. [23] performs performance analysis and purpose scheduling algorithm that suggests that if the boundary for the zones are kept adaptive then the overall performance of the network can be enhanced notably. In support of this another study by Rajya Lakshmi et al. [28] improves the performance of MMR WiMAX networks and maintains the QoS flow requirements by using adaptive Zone size based on channel quality of each node. But if the number of subscribers increases with more variable channel conditions the purposed method will be expensive to use and may affect the overall performance. Even the power mode can be used for the performance improvement in the overall network as shown by the research by Paul A. et al. [24]; exploit sleep mode parameters of IEEE $802.16 \mathrm{j}$ to control or minimize the energy needs of the mobile node. They propose a scheme called energy saving centric uplink scheduling ESCS. This scheme does bandwidth allocation and sleep cycle decision algorithm. The results show that proposed ESCS provide more sleep time to the nodes, hence making them more energy efficient. Interference is the major constraint for the wide spread shared resources in WiMAX network among the devices and become challenge for the researchers. Therefore, resource allocation and sharing become an issue. In Mahb. et al develop a greedy centralized algorithm handle this issue. Another concept introduce relays in the network comes into the lime light. Initially relays were used to extend the network coverage. But IEEE 802.16j made relays more useful in the context of improving the performance and QoS of the network.

The main question is do the relays in network can improve the overall network performance or otherwise? For this research get focus on this aspect like study by Deb S. et al. [13] demonstrated the enhancement in throughput and range extension using relays at MAC layer. The results show an improvement in the median throughput of about $25 \%$. But their analysis addresses only downlink scheduling however uplink results can be obtained similarly. Also, the relay selection for the respective subscriber remains unanswered in this work. A similar study by Genc V. et al. [15] shows that introduction of relays only improves throughput of approximately half of the coverage area of base station (BS).

Next question is, will the relay placement and selection play any role in the overall network performance. First, if we consider the relay placement lot of work shows the importance of relay placement in the context of the network overall performance like work by Chang C. Y. et al. [26] purposes a relay placement mechanism (RPM) that enhances the overall network capacity. Simulation results shows improvement in throughput and delays while maintaining a satisfactory level QoS. Also, performance evaluation in MMR networks is carried out by Ge Y. et al. [19] and they show that optimal relay selection can enhance the end-to-end throughput up to approximately $49 \%$. Further Chang C. Y. et al. in [21] propose a relay deployment algorithm with an objective to minimize the hardware cost. Result shows improvement in the time slots allocation when relays are optimally placed but still compromise regarding hardware cost. In Chang J. Y. et al. in [27] proposed relay placement in order to improve network performance by minimizing the budget using rotational clustering algorithm. The results of the purpose scheme are compared to the RSPS and RPCC schemes. The average throughput and coverage ratio show profound improvement.

Work done by Arthi M. et al. [25] mainly focuses on the IEEE 802.16j network RS placement. The author emphasis the need of proper placement of RS in the network because improper placement may introduce multitude of issues like SNR in case of densely use of RS nodes, transmission delay in case of long spread RS nodes, Coverage holes, path selection in case of more than one option available for any node finally the link overloading such as many nodes requesting communication from one RS node. The paper selects the candidate positions for the RS using throughput-oriented method. Then develop an optimized model for the deployment of the RS in the network in order to minimize the overall budget. The model simulation result shows quite improvement in the capacity of the network. Especially when the BS an RS distance increases till a point where one can get the maximum throughput. These points become the deployment place for the RS. Relay selection is also important as the relay placement.

Relay selection plays very important role in enhancing the overall network performance as shown by the study Sreng V. et al. [7] that propose relay selection strategy based on physical distance and path-loss and conclude with the results that pathloss selection scheme consistently shows superior performance in comparison to those based on distance only. However, the study only discusses the coverage extension; impact on network throughput is not considered. Researchers devise different parameters for the selection of relays, some purpose methods such as selection based on distance from BS, simplest ways in this issue. Some studies find out that SNR (signal-tonoise ratio) should be considered as a main factor for the relay selection, therefore $\mathrm{Hu} \mathrm{H}$. et al. [8] concludes that station with maximal SNR in candidate relay stations should be chosen as relay. Some selection is based on the power criteria aspect considered by the researchers for the relay selection, the algorithm proposed by Hui T. et al. [9] choose the minimal total transmission power, set P1 and P2 for the power of the relay link and access link respectively, this find the complete path and relay will be selected automatically. Some study selects the different modes of relays for the performance improvement of the network. Such as Zhu V. et al. [12] did comprehensive performance evaluations of relays in WiMAX networks and finds out that the non-transparent relay station in IEEE 802.16j network do perform better with distributed scheduling as compare to the transparent relays. But nontransparent relays are expensive and complex, and therefore most research studies are done for transparent relays which are more economical and easier to deploy. Another relay selection criterion is the mode of working principle of the relays like amplify-forward (AF) and decode and forward (DF) relays as a Study by Swain Manoj et al. [29] propose relay selection scheme based on both AF and DF types of relays. The results show that the harmonic mean is better than min max scheme as far as SNR requirements for selection of relays is concern but for bandwidth performance min max supersedes harmonic mean. However, the results are simulated keeping fixed number of subscribers. In [30], we tried to maximize network capacity by optimizing relay selection. 
In this work we consider the issues of performance and relay selection in $802.16 \mathrm{j}$ network, we propose model that makes relay selection with an objective to fairly maximize overall network capacity. Further we develop relay selection algorithm to ensure fair data rate for all the subscribers with in the range of BS. We explain the detail of our proposed system architecture and model in the next section.

\section{IEEE 802.16J Evaluation Model}

In this section, we propose an optimization model for performance evaluation of IEEE $802.16 \mathrm{j}$. Relays can be used to enhance network capacity of IEEE $802.16 \mathrm{j}$ network by increasing data rate for the subscriber and mitigating the coverage holes in the network. Simple scenario is shown in Fig. 2; it is assumed that the relay RS2 send data to SS2 and SS4 at much improved data rates due to better modulation and coding scheme causes increase in the network capacity. But here couple of questions arises; firstly, how much capacity increases in the network by using relays. Secondly what will be the relay node selection criteria? Since the IEEE802.16j left these issues vendor specific, therefore our study tries to answer these open questions. Next, we build a scenario to explain our model and demonstrate the selection criteria.

We consider a scenario in which the relay nodes are being selected based on modulation and coding scheme and we consider a system having tree topology as shown in Fig. 3. Since transparent relays assume that all the nodes can decode the framing information, therefore we assume that all the nodes are within the transmission range of base station. We have one base station and four subscribers. SS4 can communicate with the BS using four different routes; AB, CD, E and FG.

If we assume that the SS4 require fixed amount of data, for the requested demand the $\mathrm{BS}$ will allocate 30 slots via $\mathrm{AB}, 10$ slots via $C D, 20$ slots via $E$ and 15 slots via FG. The base station does this allocation based on signal to noise ratio between the base station and the nodes. In order to optimize the throughput of the network, our proposed model selects the least demanding route to fulfill the data rate requirement of SS4, which is in this case is CD with 10 slots. Rest of the routes is not considered. Since the allocation is done only for one frame at a time therefore the all the demand of the subscriber may not be fulfilled only in one frame however the allocation per frame will optimize the overall network throughput.

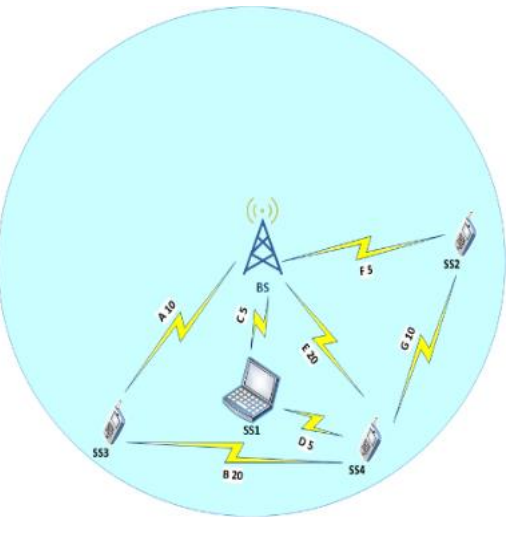

Fig. 2. Relay Network.

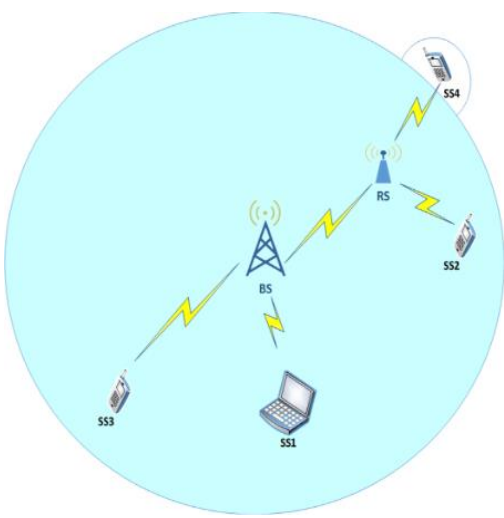

Fig. 3. Relay Scenario.

\section{A. Parameters and Notations}

We define some parameters as shown in Table $\mathrm{I}$, such as $\mathrm{S}$ is the set of all subscribers within the coverage range of the base station; $\mathrm{L}$ is set of all the links between the nodes and with the base station in the system and q is total number of slots in the downlink frame. In WiMAX TDD frame comprises of slots; which is a unit of resource occupies space both in time and frequency. $D_{s}$ is demand of each subscriber $s, M_{i j}$ is data rate per slot against a specific modulation and coding scheme for the subscriber between node $\mathrm{i}$ and $\mathrm{j}$. $\mathrm{J}$ is assumed to be base station node and finally $K_{s}$ is a set of all the nodes except the node $\mathrm{s}$ itself.

\section{B. Linear Programming Model}

We employ linear programming approach therefore first we define our decision variable $T_{S}$ which shows the number of slots allocated to each subscriber over a link. Since centralized scheduling and resource allocation is used there for BS has this responsibility. Where $\mathrm{q}$ is the number of slots allocated to the subscriber on the link $\mathrm{e}_{\mathrm{jks}}$. Here $\mathrm{j}$ is the base station, $\mathrm{k}$ belongs to the set of relays and $\mathrm{s}$ is among the set of subscribers. Therefore, the link e can be between base station and the subscriber or between the base station and the relay node.

TABLE. I. NOTATIONS

\begin{tabular}{|l|l|}
\hline Parameter & Description \\
\hline$S$ & Set of all subscribers \\
\hline$L$ & Set of all links in the system \\
\hline$q$ & Total number of slots in the DL frame \\
\hline$d_{s}$ & Demand of subscriber in set S \\
\hline$m_{i j}$ & $\begin{array}{l}\text { Data rate per slot against a specific MCS for the } \\
\text { subscriber between node i and } \mathrm{j}\end{array}$ \\
\hline$J$ & Base station node \\
\hline$k_{s}$ & Set of subscriber nodes act as relays attached to s in set S \\
\hline$e_{j s}$ & Link between base station to the subscriber \\
\hline$e_{j k}$ & Link between the base station to the relay \\
\hline$e_{k s}$ & Link between the relay to the subscriber \\
\hline
\end{tabular}




\section{Objective Function}

Objective of this proposed model is to select the relay nodes such that the overall throughput of the network is maximized. Further the data rate allocated to the subscribers should be fair, so that the network resources may not consumed by few nodes in the network. We implemented the slot wise fairness as a constraint explained below. Objective function in Eq. (1), the ${ }_{T_{s}} e_{j s} . m_{j s}, m_{j s}$ is the modulation and coding scheme between base station and the subscriber, gives us the actual data rate in the form of data bits per slot for the given subscriber over the given link. This equation calculates the data rate for all the subscriber attaches directly to the base station. While $T_{s} e_{j k_{s}} . m_{j k_{s}}$ determines the data rate between the base station and the relay node, a relay node can be any node in the network except the requesting subscriber.

We used simplex method for the maximization function, initially the function allocate slot for the subscriber over the link between the base station and the subscriber, then further allocates the slots to the relay nodes for the given subscriber over the link between base station and the relays in the same frame. The function selects the relay nodes for the given subscribers if the number of slots allocated to the subscriber in the direct link to the base station is more than the number of slots via relay nodes. The equation ${ }_{T_{s} e_{j k_{s}}{ }{ }_{j k_{s}}}$ is multiplied by two and subtracted by $T_{T_{s} e_{s_{s}} \cdot m_{k_{s}}}$ to show two hop communication.

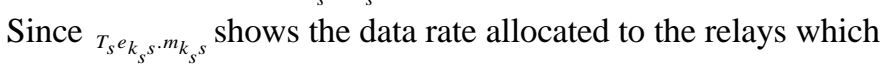
need to be subtracted to get the actual data rate of the subscribers attach to that relay. We assume centralized scheduling; therefore, base station allocates slots both for the relay and subscriber. Proposed objective is to maximize the overall data rate of the network under given constraints. These constraints are explained one by one as below:

$$
\begin{aligned}
& \sum_{s} T_{s} e_{j s} \cdot m_{j s}+2 \sum_{s} \sum_{k_{s}} T_{s} e_{j k_{s}} \cdot m_{j k_{s}}-\sum_{s} \sum_{k_{s}} T_{s} e_{k_{s}} \cdot m_{k_{s} s} \\
& j \in J, k_{s} \in K_{s}, s \in S
\end{aligned}
$$

\section{Demand Constraint}

First constraint is obvious that the actual data rate allocated to each subscriber must be equal or less than its actual demand. Since the demand of the subscriber does vary therefore to keep the system safe from abnormal allocations this constraint plays a vital role. In Eq. (2), $T_{s} e_{j s} \cdot m_{j s}$ shows data rate of the subscriber for direct link between base station and the subscriber. Similarly, $T_{s} e_{j k_{s}} \cdot m_{j k_{s}}$ shows the data rate for the subscriber over the indirect link via relay. If the subscriber attaches to the base station directly then the relay link will have Ts with zero value, which further makes the data rate insignificant. Therefore, numerically we will be getting the data rate of only one link, which in our scenario is the direct one.

$$
\begin{aligned}
& T_{s} e_{j s} \cdot m_{j s}+\sum_{k_{s}} e_{j k_{s}} \cdot m_{j k_{s}} \leq d_{s} \\
& j \in J, k_{s} \in K_{s}, s \in S
\end{aligned}
$$

\section{E. Data Preservation Constraint}

This constraint enforces the fact that amount of data received by the subscriber should be equal to the amount of data send by the base station to the relay node for the subscriber. As this is shown in Eq. (3), is the link between base station and the relay node for a subscriber. Similarly, the ekss shows the link between subscriber's relay nodes to that subscriber. In other words, it can be said that the data transmitted between the relay link and the access link for a particular subscriber should be same.

$$
\begin{aligned}
& T_{s} e_{j k_{s}} \cdot m_{j k_{s}}=T_{s} e_{k_{s} s} \cdot m_{k_{s} s} \\
& j \in J, k_{s} \in K_{s}, s \in S
\end{aligned}
$$

\section{F. Resource Constraint}

In the world of wireless data communication resources are the most precious and scarce thing. In proposed modal the resource we consider is the physical slots. Since q represent the total number of slots in a downlink frame. Therefore, it is checked that the total number of slots allocated to all the subscribers and relays should be equal or less than the total number of slots available i.e. q. In Eq. (4), ${ }_{T_{s}} e_{j s}$ represent the number of slots allocated to the subscriber over the direct link between the base station and the subscriber. $T_{s} e_{j k_{s}}$ shows the number slots allocated to the relay nodes over the link between the base station and the relay node. $T_{s} e_{k_{s} s}$ here the Ts represents the number of slots allocated to the subscriber attach to the relay ks. Summation of all the allocated slots to all the nodes over all the links should be less or equal to q.

$$
\begin{aligned}
& \sum_{s} T_{s} e_{j s}+\sum_{s} \sum_{k_{s}} T_{s} e_{j k_{s}}+\sum_{s} \sum_{k_{s}} T_{s} e_{k_{s} s} \leq q \\
& j \in J, k_{s} \in K_{s}, s \in S
\end{aligned}
$$

\section{G. Share Constraint}

In order to make our model fair, following equations are constructed. Here in Eq. (5), $\mathrm{u}$ is the total number of subscribers in a network. $(q / u) \cdot m_{j s}$ gives average data rate for each subscriber. $(q / u) \cdot m_{j s}-d_{s}$ this equation checks that if the demand of subscriber is more than the average data rate available for it or vice versa. In case of more demand Eq. (5) will be executed. Eq. (6) ensures that each subscriber should get at least average data rate or more if possible. In the next section we compare our model with fixed WiMAX. The simulation results show substantial improvement in the overall network throughput. These results with given scenarios are further elaborated below:

$$
\begin{aligned}
& (q / u) \cdot m_{j s}-d_{s} \geq 0 \\
& j \in J, s \in S \\
& T_{s} e_{j s} \cdot m_{j s}+\sum_{k_{s}} T_{s} e_{j k_{s}} \cdot m_{j k_{s}}-d_{s}=0 \\
& j \in J, k_{s} \in K_{s}, s \in S
\end{aligned}
$$


Where

$$
(q / u) \cdot m_{j s}-d_{s} \geq 0, \quad j \in J, s \in S
$$

\section{RELAy SELECTION ALGORITHM}

In the proposed relay selection algorithm, all the parameters are defined in Table II. In this when our objective function got maximized the slots over all the links are assigned for a subscriber. First the slots required for the SS over direct link between BS and the SS are allocated then slots for the SS and all the other SS's other than BS are assigned. The algorithm selects link with minimum number of slots required to full fill the demand of the subscriber. This link can be direct between the BS and SS or BS to RS then to SS depend upon the number of slots required. This algorithm optimizes the slots assignment to all subscribers such that every user may get a minimum number of useful resources.

We use for loop since the function will iterate for each node in the network. The function first part that is $T_{a s} \cdot m_{j s}$ assign slots to the nodes directly attached to the base station. While second part of the function $2\left(T_{r s} . m_{j k_{s}}\right)-T_{k s} \cdot m_{k_{s} s}$ assign slots to the nodes that require relays for the communication. After that $T_{a s} . m_{j s}+T_{r s} . m_{j k_{s}}>d_{s}$ is a demand constraint that enforce the check that the total resource allocated to the node must not increase the demand requested by the node. Next $T_{r s} \cdot m_{j k_{s}} \neq T_{k s} \cdot m_{k_{s} s}$ ensure data integrity it means that the data send by the base station should be equal to the data received by the node. Following this is a resource constraint $\left(T_{a s} \cdot m_{j s}+T_{r s} \cdot m_{j k_{s}}+T_{k s} \cdot m_{k_{s} s}\right)>q$ states that the total slots assign to all the nodes must not surpass the given maximum number of slots. Finally the share constraint insert fairness attribute in the algorithm $T_{a s} \cdot m_{j s}+T_{r s} \cdot m_{j k_{s}}-d_{s} \neq 0$ this equation forces the algorithm to assign some average resource to the node. Next section will discuss the results we obtain from this algorithm.

TABLE. II. ALGORITHM PARAMETERS

\begin{tabular}{|l|l|}
\hline Parameter & Description \\
\hline$J$ & Base Station \\
\hline$S$ & Set of all nodes \\
\hline$k_{s}$ & Set of all nodes except s \\
\hline$m_{i j}$ & Set of all MCS between Base station and nodes S \\
\hline$m_{j k_{s}}$ & Set of all MCS between Base station and nodes Ks \\
\hline$m_{k_{s} s}$ & Set of all MCS between nodes S and Ks \\
\hline$d_{s}$ & Demand of node s \\
\hline$T_{a s}$ & number of nodes assign over the link BS and s node \\
\hline$T_{r s}$ & number of nodes assign over the link BS and Ks node \\
\hline$T_{a k}$ & number of nodes assign over the link Ks and s node \\
\hline
\end{tabular}

Algorithm 1. Relay Selection Algorithm

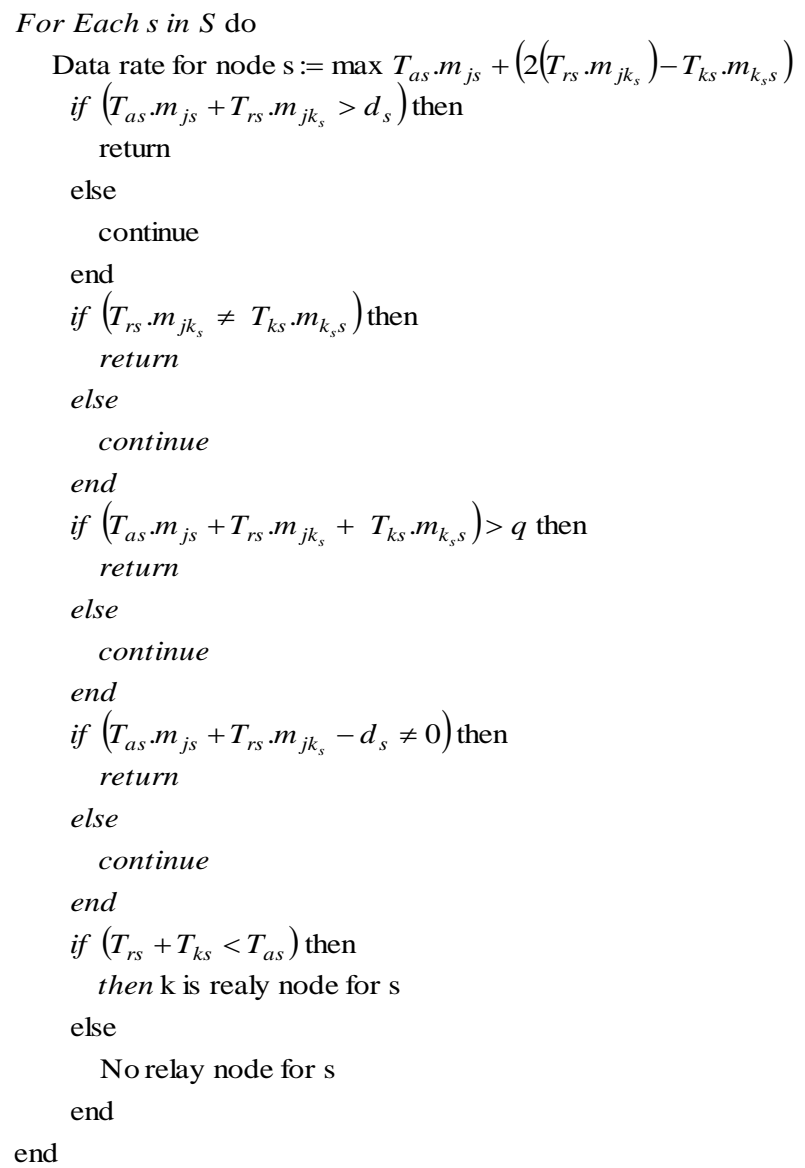

\section{RESUltS AND DisCUSSION}

For the simulation purpose we employed tree-based topology with BS at the root and centralized scheduling is assumed. Also, nodes only two hops away from the BS are taken under consideration. Since more than two hops have capacity issues depend upon subscriber density. We create different downlink scenarios to test the effectiveness of our proposed model. The scenario we assume is such that on OFDMA PHY using frequency $3.5 \mathrm{GHz}, 20 \mathrm{MHz}$ channel bandwidth, size is 2048 FFT, contain 1440 data subcarrier and $1 / 8$ cyclic prefix. In addition to this other parameter like noise figure and thermal noise at transmitter are adjusted at $13 \mathrm{db}$ and $-174 \mathrm{dbm}$ respectively. The frame size is assumed to be $20 \mathrm{~ms}$. The DL sub frame is about uses 50 of the total frame sizes.

Major parameter that we take under consideration for analysis purpose is SNR [signal to noise ratio]. SNR value determines the quality of a link ranges from 1-26, if its value is high then link is good and can transmit data at higher rate because of better modulation and coding scheme and vice versa. Our focus in this simulation is to cover all the possible scenarios that come across in networks. Table III shows the quantitative values of SNR against which the respective modulation and coding scheme is selected. In this we see that as the values of SNR increases, the better MCS are mapped against them. 
TABLE. III. SignAl to Noise RAtio

\begin{tabular}{|l|l|l|}
\hline Modulation & Coding Scheme & Receiver (SNR) \\
\hline BPSK & $1 / 2$ & 3.0 \\
\hline QPSK & $1 / 2$ & 6.0 \\
\hline QPSK & $3 / 4$ & 8.5 \\
\hline $16-Q A M$ & $1 / 2$ & 11.5 \\
\hline 16-QAM & $3 / 4$ & 15.0 \\
\hline 64-QAM & $2 / 3$ & 19.0 \\
\hline 64-QAM & $3 / 4$ & 21.0 \\
\hline
\end{tabular}

\section{A. Scenario 1: Random SNR for All Subscribers}

First case we consider random value of SNR for all the subscribers as shown in Fig. 4. The graph shows throughput, at $y$-axis, achieved for different number of subscribers, shown at $\mathrm{x}$-axis. In this scenario, we consider thirty subscribers against different demands. As we increase the number of users, we also vary the demand for the subscriber, but this demand remains same for all the users under consideration. The results show great increase initially as the users were less and demand was nominal too, as compare to the WiMAX network without relays. However, as the user demand increases the percentage decreases but remains up to $66 \%$. Scenario in which we consider the demand of the entire subscriber fixed, and by only varying the SNR for different subscribers, we can see that the performance of our model represented as $\mathrm{J}$ is giving up to $66 \%$ increase compare to fixed WiMAX.

\section{B. Scenario 2: 90\% Subscribers with Excellent SNR}

In another experiment we test a scenario in which $90 \%$ of subscribers are assumed to have SNR 15-26db, a much better channel condition. While the rest of $10 \%$ has poor channel condition or have low values of SNR. Initially we increase the number of users at the same rate as described in scenario 1 . In addition to this the demand increases simultaneously. Only difference here is that the SNR is not random, but we have fixed the SNR to certain percentage of users.

Here as shown in Fig. 5, model is still able to get $8 \%$ increase of network throughput as compare to the WiMAX without relays. Since in this case if the BS coverage is good then the relays will not make much difference, however in cases like shadowing or dark spots our model still prove to be much more efficient the plain WiMAX network. The results again show that model performance is $8 \%$ more than the $\mathrm{S}$.

\section{Scenario 3: 10\% Subscribers with Excellent SNR}

Now in this experiment we swap the scenario and only $10 \%$ of subscriber have best SNR values between $15 \mathrm{db}$ to $26 \mathrm{db}$ while the rest of subscriber have SNR values between $0 \mathrm{db}$ to $14 \mathrm{db}$. The results shown in Fig. 6 are quite convincing. This time model output shows $63 \%$ improvement in the context of throughput as compared to WiMAX without relays. Here again we have same number of subscribers which we increase gradually with different demands. Here we see the true benefit of relays in the network when the BS coverage is not good then our model can play significant role in improving the overall throughput of the network by giving alternate routes to the subscribers with low or poor SNR values.

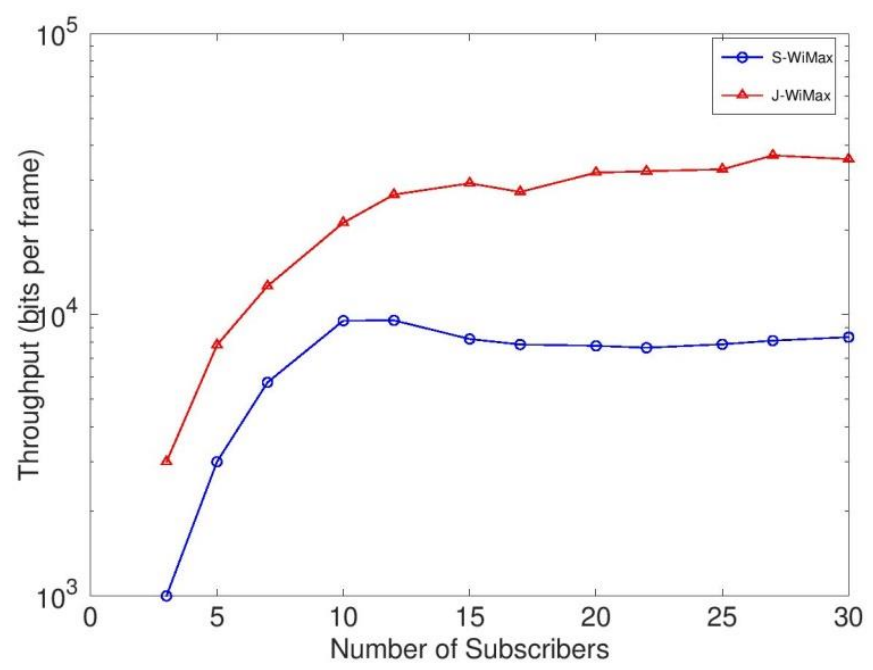

Fig. 4. Random SNR for all Subscribers.

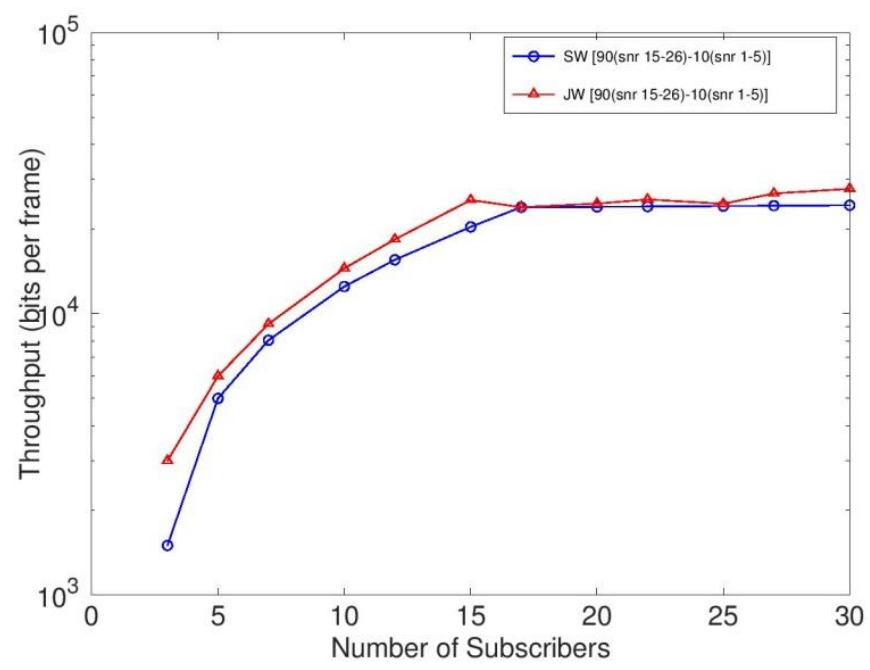

Fig. 5. 90\% Subscribers with Excellent SNR.

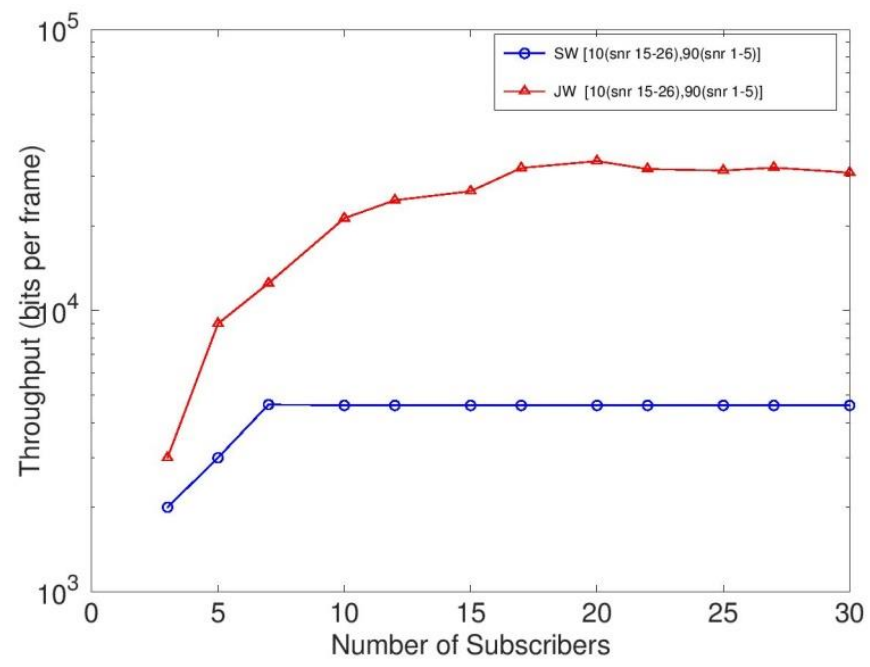

Fig. 6. $10 \%$ Subscribers with Excellent SNR. 


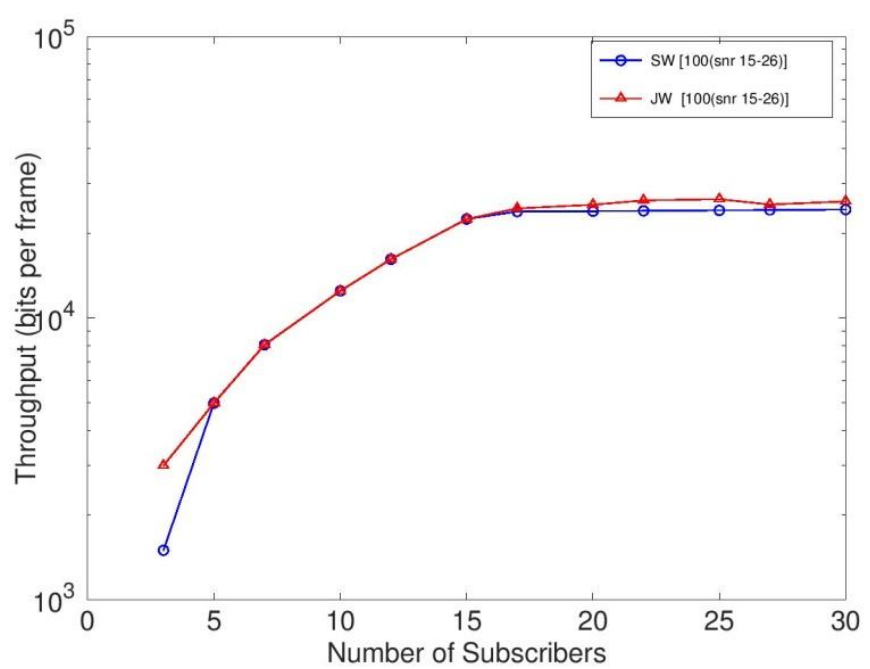

Fig. 7. $100 \%$ Subscribers with Excellent SNR.

\section{Scenario 4: 100\% Subscribers with Excellent SNR}

Now we start to check for the extreme cases, we gave all the subscribers best SNR values $15-26 \mathrm{db}$, again we increase the subscribers and demand as we did in the previous experiments. Here the results shown in Fig. 7 tell us that we can have throughput improvement by $4 \%$ using our model as compared to the WiMAX without relays. Again, we see that if we assume the network coverage to be best for all the subscribers then again the rule of relays remain quite trivial, and the results are more or less like the previous experiment as shown in Fig. 5.

\section{E. Scenario 5: $100 \%$ Subscribers with Poor SNR}

This scenario considers all subscribers with lowest SNR values 1-5. In this scenario again we increase the number of subscribers and the demand gradually, but the results shown in Fig. 8 are not much convincing. In this the results shows that if there are small number of subscribers then our model does perform much better than WiMAX without relays, however as the number of subscribers increases then there is not much difference between the WiMAX network using our model and the WiMAX network without the relays. Again, this shows that in extreme scenarios the behavior of network throughput is not much changed using relays. But such scenarios are quite difficult to get established. Even in these extreme scenarios our model still extracts some efficiency where ever and whenever is possible, therefore it is much better to use relays where ever it is feasible.

\section{F. Scenario 6: 100\% Subscribers with Good SNR}

In another scenario we assume good channel conditions for all the subscribers. Now this is somewhat more realistic scenario. Here again, the number of users is the same and increased gradually while the demand got varied too. The SNR for all the subscriber is between $10-20 \mathrm{db}$ which automatically end up having much improved modulation and coding scheme. The results in Fig. 9 again show that the results shown by our model against the simple WiMAX without relays are $22 \%$ better. And the results got better as the number of users increases and demand of the user increases.

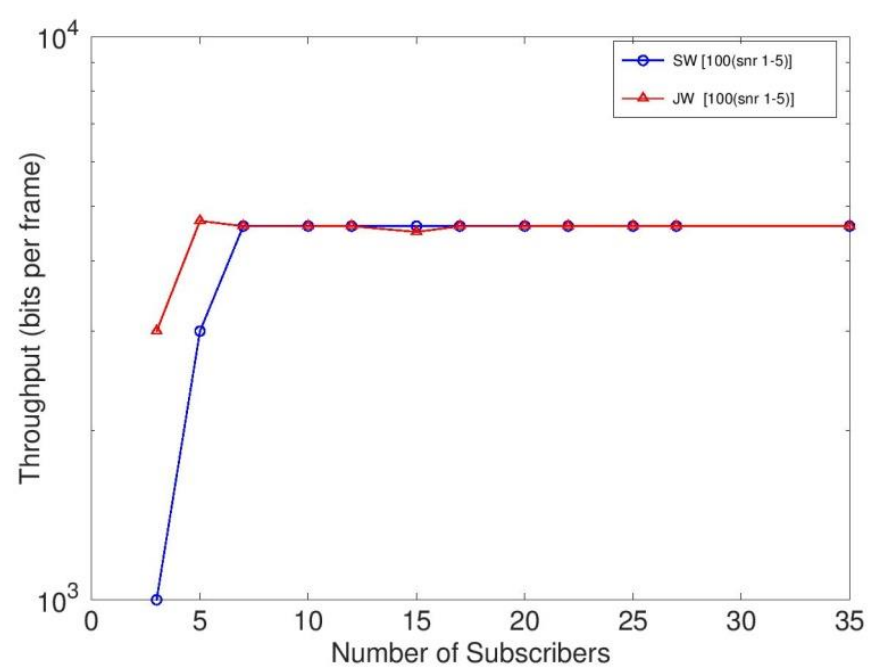

Fig. 8. 100\% Subscribers with Poor SNR.

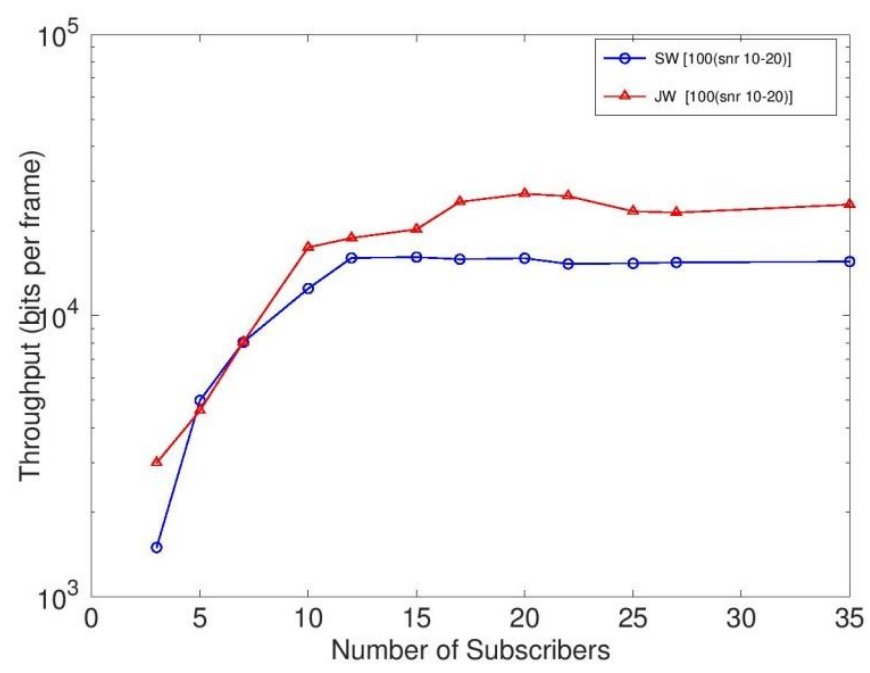

Fig. 9. 100\% Subscribers with Good SNR.

\section{CONCLUSION}

This work proposes a linear model for the IEEE 802.16j network to study the overall network capacity gains. The study helps to understand the performance effects after deploying the relays in WiMAX network. Mainly, multiple aspects of network are taken under consideration like performance changes under certain conditions and relay placements implication on overall network capacity. The study developed a linear modal for capacity evaluation and relay placement algorithm for its analysis. The results show comprehensive increase in throughput up to $66 \%$ in overall network capacity using relays in a fixed WiMAX network. In our future work, we are currently working to incorporate relay selection while satisfying quality of service (QoS). Different aspects of QoS can be explored to see how much difference it will create compared to other schemes.

\section{REFERENCES}

[1] IEEE 802.16 Working Group. Ieee standard for local and metropolitan area networks-part 16: Air interface for fixed broad-band wireless access systems. IEEE Std. 802.16-2004. 2004. 
[2] IEEE LAN/MAN Standards Committee. IEEE Standard for local and metropolitan area networks Part 16: Air interface for fixed and mobile broadband wireless access systems amendment 2: Physical and medium access control layers for combined fixed and mobile operation in licensed bands and corrigendum 1. IEEE Std 802.16 e-2005. 2006.

[3] IEEE 802.16 Working Group. Ieee standard for local and metropolitan area networks-part 16: Air interface for fixed broad-band wireless access systems. IEEE Std. 802.16-2004. 2004.

[4] IEEE 802.16 Working Group. IEEE standard for local and metropolitan area networks, part 16: Air interface for broadband wireless access systems, amendment 1: Multi-hop relay specification. IEEE Standard 802.16 j-2009. 2009.

[5] Pareit D, Lannoo B, Moerman I, Demeester P. The history of WiMAX: A complete survey of the evolution in certification and standardization for IEEE 802.16 and WiMAX. IEEE Communications Surveys \& Tutorials. 2011 Oct 13;14(4):1183-211.

[6] Pabst, Ralf, Bernhard H. Walke, Daniel C. Schultz, Patrick Herhold, Halim Yanikomeroglu, Sayandev Mukherjee, Harish Viswanathan et al. "Relay-based deployment concepts for wireless and mobile broadband radio." IEEE Communications Magazine 42, no. 9 (2004): 80-89.

[7] Sreng, Van, Halim Yanikomeroglu, and David D. Falconer. "Relayer selection strategies in cellular networks with peer-to-peer relaying." In 2003 IEEE 58th Vehicular Technology Conference. VTC 2003-Fall (IEEE Cat. No. 03CH37484), vol. 3, pp. 1949-1953. IEEE, 2003.

[8] Hu, Huining, Halim Yanikomeroglu, David D. Falconer, and Shalini Periyalwar. "Range extension without capacity penalty in cellular networks with digital fixed relays." In IEEE Global Telecommunications Conference, 2004. GLOBECOM'04., vol. 5, pp. 3053-3057. IEEE, 2004.

[9] Hui, Tian, Gu Xuelin, and Zhang Ping. "The impact of relaying strategies on the performance in cellular system." In IEEE International Symposium on Communications and Information Technology, 2005. ISCIT 2005., vol. 2, pp. 1404-1407. IEEE, 2005.

[10] Bonald, Thomas, Laurent Massoulié, Alexandre Proutiere, and Jorma Virtamo. "A queueing analysis of max-min fairness, proportional fairness and balanced fairness." Queueing systems 53, no. 1-2 (2006): 65-84.

[11] Shrestha, Deepesh Man, Sung-Hee Lee, Sung-Chan Kim, and YoungBae Ko. "New approaches for relay selection in IEEE 802.16 mobile multi-hop relay networks." In European Conference on Parallel Processing, pp. 950-959. Springer, Berlin, Heidelberg, 2007.

[12] Zhu VM, Viorel VD. Multihop relay extension for WiMAX networksoverview and benefits of IEEE $802.16 \mathrm{j}$ standard. Fujitsu Sci. Tech. J. 2008 Jul;44(3):292-302.

[13] Deb, Supratim, Vivek Mhatre, and Venkatesh Ramaiyan. "WiMAX relay networks: opportunistic scheduling to exploit multiuser diversity and frequency selectivity." In Proceedings of the 14th ACM international conference on Mobile computing and networking, pp. 163174. ACM, 2008.

[14] Ann, Sojeong, Kyung Geun Lee, and Hyung Seok Kim. "A path selection method in IEEE $802.16 \mathrm{j}$ mobile multi-hop relay networks." In 2008 Second International Conference on Sensor Technologies and Applications (sensorcomm 2008), pp. 808-812. IEEE, 2008.

[15] Genc, Vasken, Seán Murphy, and John Murphy. "Performance analysis of transparent relays in 802.16 j MMR networks." In 2008 6th International Symposium on Modeling and Optimization in Mobile, $\mathrm{Ad}$ Hoc, and Wireless Networks and Workshops, pp. 273-281. IEEE, 2008.
[16] Genc, Vasken, Sean Murphy, and John Murphy. "An interference-aware analytical model for performance analysis of transparent mode $802.16 \mathrm{j}$ systems." In 2008 IEEE Globecom Workshops, pp. 1-6. IEEE, 2008.

[17] Genc, Vasken, Seán Murphy, and John Murphy. "Analysis of transparent mode IEEE $802.16 \mathrm{j}$ system performance with varying numbers of relays and associated transmit power." In 2009 IEEE Wireless Communications and Networking Conference, pp. 1-6. IEEE, 2009.

[18] Li, Dandan, and Hao Jin. "Relay selection in two-hop IEEE 802.16 Mobile Multi-hop Relay networks." In 2009 First International Workshop on Education Technology and Computer Science, vol. 2, pp. 1007-1011. IEEE, 2009.

[19] Ge, Yu, Su Wen, and Yew-Hock Ang. "Analysis of optimal relay selection in IEEE 802.16 multihop relay networks." In 2009 IEEE Wireless Communications and Networking Conference, pp. 1-6. IEEE, 2009.

[20] Mach, Pavel, Robert Bestak, and Zdenek Becvar. "Optimization of association procedure in WiMAX networks with relay stations." Telecommunication Systems 52, no. 3, 2013.

[21] Chang, Chih-Yung, and Ming-Hsien Li. "A placement mechanism for relay stations in 802.16 j WiMAX networks." Wireless networks 20, no. 2, 2014.

[22] Chang, Jau-Yang, and Ya-Sian Lin. "A clustering deployment scheme for base stations and relay stations in multi-hop relay networks." Computers \& Electrical Engineering 40, no. 2, 2014.

[23] Liu, I-Hsien, Chuan-Gang Liu, Chien-Tung Lu, Yi-Tsen Kuo, and JungShian Li. "A multi-hop resource scheduling algorithm for IEEE $802.16 \mathrm{j}$ relay networks." Computer Networks 67, 2014.

[24] Paul, A., Anagha, P., and Umaparvathi, M. "Energy Efficient Scheduling For Wimax Network", International Journal of Software \& Hardware Research in Engineering, 3(3), 2015.

[25] Arthi, M., Jimy Jose Joy, P. Arulmozhivarman, and K. Vinoth Babu. "An efficient relay station deployment scheme based on the coverage and budget constraints in multi-hop relay networks." In 2015 International Conference on Communications and Signal Processing (ICCSP), pp. 0124-0128. IEEE, 2015.

[26] Chang, Chih-Yung, Chao-Tsun Chang, Tzu-Chia Wang, and MingHsien Li. "Throughput-enhanced relay placement mechanism in WiMAX 802.16 j multihop relay networks." IEEE systems journal 9, no. 3, 2014.

[27] Chang, Jau-Yang, and Yun-Wei Chen. "A relay station deployment scheme with a rotational clustering algorithm for multi-hop relay networks." In 2016 International Conference on System Science and Engineering (ICSSE), pp. 1-4. IEEE, 2016.

[28] Lakshmi, L. Rajya. "Adaptive Zone Size Selection Method for IEEE 802.16 j Mobile Multihop Relay Networks." Wireless Personal Communications 97, no. 4, 2017.

[29] Swain, Chaudhuri Manoj Kumar, and Susmita Das. "Study and Impact of Relay Selection Schemes on Performance of an IEEE 802.16 j Mobile Multihop Relay (MMR) WiMAX Network." In Progress in Intelligent Computing Techniques: Theory, Practice, and Applications, pp. 491499. Springer, Singapore, 2018.

[30] N. Mazhar, "802.16j Network Performance Analysis and Relay Selection" MS. dissertation, Dept. Computing, SEECS, NUST Univ., Islamabad, Pakistan, 2011. 